Josip Ivankić

HEP Trade LTD

josip.ivankic@hep.hr

\title{
Deni Ćetković
}

HEP-Distribution system operator

deni.cetkovic@hep.hr

prof. dr. sc. Dario Matika

Faculty of Engineering, University of Rijeka dmatika@riteh.hr

\section{Maintenance of Filter at The Gas Turbine Compressor Intake and Electric Transformer Connector Based on Operational Reliability}

\section{SUMMARY}

The article analyzes the operational reliability of filter on the gas turbine compressor intake and the operational reliability of electric transformer connector. Empirical data (statistical sample) were collected to determine the failure density function $\mathrm{f}(\mathrm{t})$, the hazard function ? $(\mathrm{t})$, and the expected value of mean time to failure MTTF. The numerical model was created in the Minitab 19 software tool. The Anderson-Darling test was used to accept or reject the hypothesi

\section{KEY WORDS}

compressor, filter, probability distribution, reliability

\section{INTRODUCTION}

1. INTRODUCTION

Reliability is defined as a measure of a device's ability to operate without failure, and it mathematically predicts the behavior of a system or device under expected operating conditions [4]. The reliability of a system is divided into structural and operational. Structural reliability refers to the construction of the system, ie it is determined mathematically by the manufacturer, while operational reliability is determined from the operation of the system, and on the basis of empirical data [2]

The first part of the paper presents an analysis of the operational reliability of the filter on the gas turbine compressor intake. The intake air filtration system is crucial for the successful operation of a gas turbine. The filtration system protects the gas turbine from harmful impurities in the outside air, which can lead to problems such as output power fluctuations, erosion, dirt, and corrosion. The main cause of the problem is the dirt of the compressor. In such cases, operators often use compressor washing as a measure, for a quick repair and, and to restore output power and efficiency. The success of this measure is short-lived because cleaning simply washes away contaminants from the front blades to the inner blades of the turbine. Turning off the turbine allows for significantly more efficient washing, but causes costly downtime. In any case, the loss of production capacity is reversed due to contaminants that are continuously generated and damage the blades and other components. If the compressor suction filter is damaged or dirty, the power plant operation must be stopped and the filter replaced. The aim of this paper is to develop a model of preventive maintenance, in order to be able to predict the potential failure of the filter based on empirical data and to prevent the sudden exit of the power plant from the operation. Filters can also be purchased in advance and kept in stock, thus reducing the logistical maintenance time, which will speed up the return of the power plant to operation, or reduce financial losses due to production downtime.

The second part of the paper presents an analysis of the operational reliability of electric transformer medium voltage connector in the transformer substation system 20/0.4 kV with installed power of 630 kVA. Due to prolonged exposure to high current flows, over time, the cable head insulation may break. In this case the protection device will disconnect transformer from the distribution system and the consumers are left without electricity until the fault is repaired. The aim of this paper is to develop a model of preventive maintenance, in order to be able to predict the potential failure 
of the connector in transformer station based on empirical data and to prevent the sudden electric power failure that would left consumers without electricity and cause financial losses to electric energy seller. The main objective is to determine the optimal point for preventive replacement of the transformer connector subsystem.

\section{MATHEMATICAL MODEL OF RELIABILITY ANALYSIS OF THE FILTER OF THE GAS TURBINE COMPRESSOR INTAKE}

Researching the reliability of the technical composition is very important because in this way failures can be predicted, and financial losses resulting from the failure of the system or a device can be reduced [7]. The mathematical model of reliability analysis is determined with two functions, namely the reliability function $R(t)$, and the failure intensity function ? $(t)$ [8], [9]. Reliability expresses the numerical probability of a device operating without failure during a certain time interval and under the operating conditions for which the device is intended, while the failure intensity function shows how the failure intensity changes during the life cycle of the system or device [3]. The reliability of a system is calculated according to the formula:

$$
R(t)=1-F(t)=1-\int_{0}^{t} f(t) d t
$$

where $F(t)$ - a function of unreliability, and $f(t)$ - function of failure probability density.

Another important quantity is the expected time to failure (MTTF), which represents the average time that the system or device works before the failure, and is calculated according to the formula [2], [13]:

$$
M T T F=\int_{0}^{\infty} R(t) d t
$$

In order to determine the probability distribution function that describes the collected fault data, the following hypothesis is set:

$\mathrm{H}_{0}{ }^{0}$ - the statistical sample is equal to the three-parameter Weibull distribution at a significance level of 0,05

$\mathrm{H}^{1}$ - the statistical sample is not equal to the three-parameter Weibull distribution at a significance level of 0,05

To accept or reject the hypothesis, the Anderson-Darling statistical test was used, which was made using the Minitab 19 program. The AndersonDarling test is a statistical test that checks for a given sample of data on how well they match the selected theoretical probability distribution [14]. Anderson-Darling statistics (AD *) Anderson-Darling statistics measure how well the data track a particular distribution [4]. For a given data set and distribution, the more appropriate the distribution for the data, the smaller the statistics will be.

\section{MATHEMATICAL MODEL OF RELIABILITY ANALYSIS OF TRANSFORMER CONNECTOR SUBSYSTEM}

The main objective is to determine the optimal point for preventive replacement of the transformer connector subsystem. The optimal point for preventive replacement of the transformer connector subsystem should be determined taking into account the minimum costs of preventive maintenance $c_{p}$, costs of corrective maintenance ck, failure intensity function ? ${ }_{k, R e}(t)$, preventive maintenance intensity function $\lambda_{p, R e}(t)$ and operational reliability function $R_{e}(t)$ [1], [15].

The function of relative maintenance costs is described by the following relation:

$$
c_{u, R e, r e l}\left(T_{R}\right)=\frac{c_{p, R e, r e l}}{100} \cdot \lambda_{p, R e l}(t)+\frac{c_{k, R e, r e l}}{100} \cdot \lambda_{p, R e l}(t)
$$

where $\mathrm{c}_{\mathrm{p} \text {,Rerel }} \mathrm{i} \mathrm{ck}_{\mathrm{Re}, \text { rel }}$ represent the relative costs of preventive and corrective maintenance.

Optimal time for replacement of the cable head subsystem could be obtained by minimizing relation (3).

Relative costs of preventive and corrective maintenance could be obtained by following relations:

$$
\begin{aligned}
& c_{p, \text { Re,rel }}=\frac{c_{p}}{c_{p}+c_{k}} \cdot 100 \\
& c_{k, \text { Re,rel }}=\frac{c_{k}}{c_{p}+c_{k}} \cdot 100
\end{aligned}
$$

The costs of corrective maintenance are described with the following relation:

$$
c_{k}=c_{M}+c_{R} \cdot E_{K A}(T)+c_{N} \cdot E_{K}(T)
$$

where $c_{M}$ is estimated material cost $(3700 \mathrm{kn}), \mathrm{c}_{\mathrm{P}}$ is cost of labor $(210 \mathrm{kn} / \mathrm{h})$ ,$c_{N}$ is estimated cost due to undelivered electricity $(348 \mathrm{kn} / \mathrm{h}), E_{K}(T)$ is time needed for corrective maintenance and $E_{K A}(T)$ is active time needed for corrective maintenance [12].

The costs of preventive maintenance are described with the following relation:

$$
c_{p}=c_{M}+c_{R} \cdot E_{K A}(T)
$$

The function of preventive replacement of a part of the transformer connector subsystem is described by the following relation:

$$
\lambda_{p, R e l}(t)=\frac{1}{T_{R}}
$$

The failure intensity function of the transformer connector subsystem is described by the following relation:

$$
\lambda_{k, R e l}(t)=\frac{T_{R}-\int_{0}^{T_{R}} R_{e}(t) d t}{T_{R} \cdot \int_{0}^{T_{R}} R_{e}(t) d t}
$$

The operational reliability of $R_{e}(t)$ is described by the following relationship:

$$
R_{e}(t)=1-\int_{0}^{t} f_{e}(t) d t
$$

where $f_{e}(t)$ is fault probability function. 


\section{RESEARCH RESULTS}

\subsection{RESEARCH RESULTS OF THE OF}

RELIABILITY ANALYSIS OF THE FILTER OF THE GAS TURBINE COMPRESSOR INTAKE

The empirical data used in the analysis are given in the following table:

Table I. Operating time between failures and ordinal number of failures

\begin{tabular}{|c|c|}
\hline Ordinal number of the fault & Operating time to failure $[\mathrm{h}]$ \\
\hline 1 & 35 \\
\hline 2 & 44 \\
\hline 3 & 114 \\
\hline 4 & 92 \\
\hline 5 & 163 \\
\hline 6 & 113 \\
\hline 7 & 115 \\
\hline 8 & 197 \\
\hline 9 & 44 \\
\hline 10 & 258 \\
\hline 11 & 532 \\
\hline 12 & 114 \\
\hline
\end{tabular}

The results of the research show that the collected empirical data best follow the log-logistic function, ie for it, the value of the Anderson-Darling test is the lowest, although the three-parameter Weibull distribution and the three-parameter log-normal distribution are very close to the AndersonDarling test values. Therefore, the hypothesis is rejected, and the hypothesis is accepted. In reliability theory, the Weibull distribution is the most commonly used distribution because it can be applied to model many different data sets, that is, it is very flexible [1], [10]. The log-normal distribution is typically used for model system or device failures caused by corrosion or chemical reactions. Log-logistic distribution is used in various fields, such as survival analysis, hydrology, economics, etc [11]. Also, loglogistic distribution well approximates normal and log-normal distribution. Although the values of the Anderson-Darling test are close for these three distributions, the log-logistic distribution was selected, and it is used for further calculations.

\section{Distribution ID Plot: C2}

\section{Goodness-of-Fit}

\begin{tabular}{lrr} 
Distribution & $\begin{array}{r}\text { Anderson-Darling } \\
\text { (adj) }\end{array}$ & $\begin{array}{r}\text { Correlation } \\
\text { Coefficient }\end{array}$ \\
\hline Weibull & 1,743 & 0,947 \\
Lognormal & 1,320 & 0,972 \\
Exponential & 1,655 & * \\
Loglogistic & 1,292 & 0,974 \\
3-Parameter Weibull & 1,358 & 0,972 \\
3-Parameter Lognormal & 1,328 & 0,973 \\
2-Parameter Exponential & 1,519 & * \\
3-Parameter Loglogistic & 1,309 & 0,974 \\
Smallest Extreme Value & 6,273 & 0,769 \\
Normal & 2,143 & 0,851 \\
Logistic & 1,849 & 0,859
\end{tabular}

Figure 1. results of the Anderson-Darling test for the collected empirical data

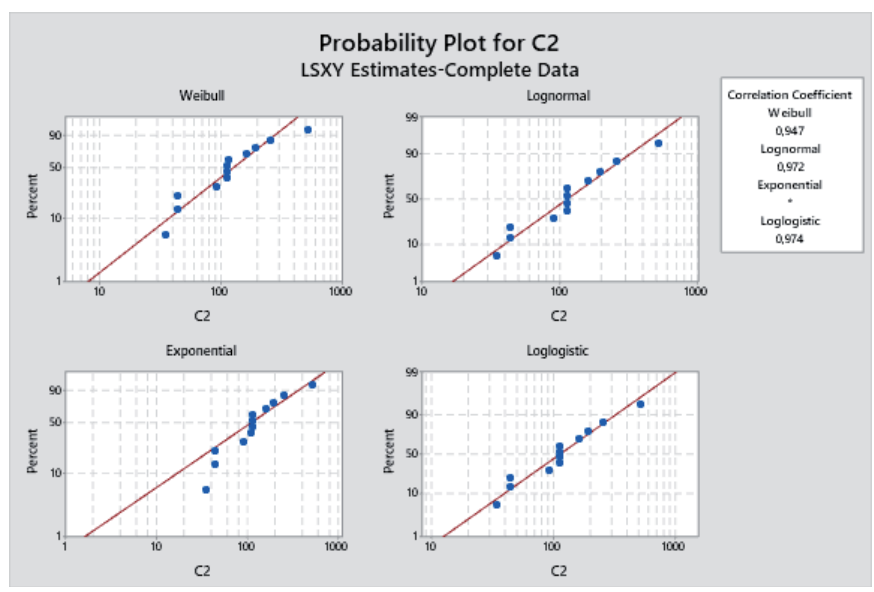

Figure 2. Hypothesis testing

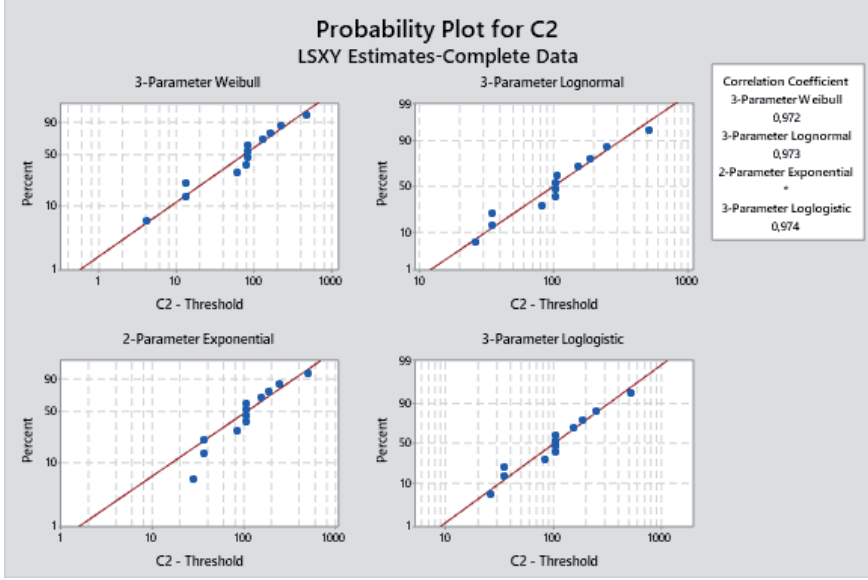

Figure 3. Hypothesis testing

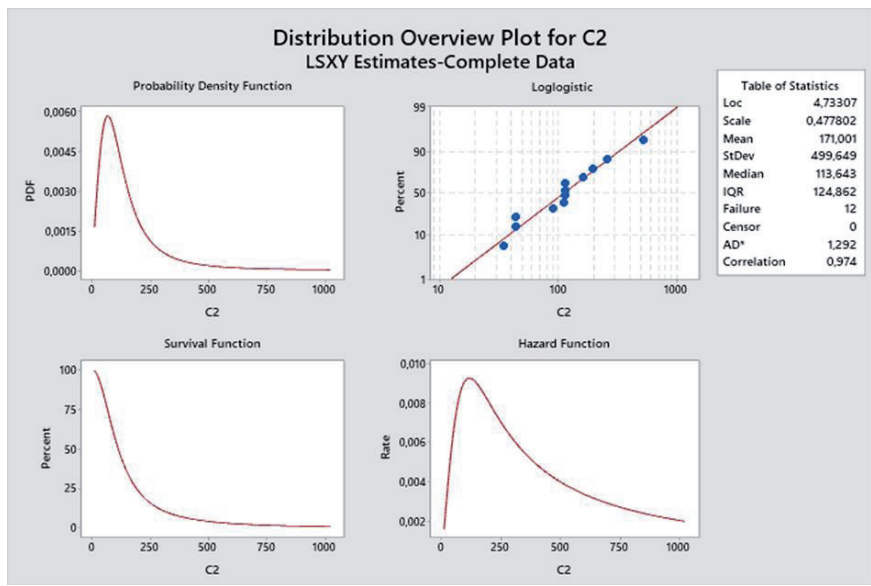

Figure 4. Hypothesis testing

The density function of the log-logistic distribution is given by the formula:

$$
f(t)=\frac{e^{z}}{\sigma t\left(1+e^{z}\right)^{2}}
$$

where:

$$
z=\frac{t^{\prime}-\mu}{\sigma}
$$




$$
t^{\prime}=\ln (t)
$$

The values of these parameters were selected based on the results obtained by analysis, and they are as follows: $\mu=0,4778$; $\sigma=4,733$; $\mathrm{t}=$ $171 \mathrm{~h}$.

It follows that: $t=\ln (171)=5,142, z=0,9855$.

From the results of the analysis it can be read that the expected failure time is: $\mathrm{MTTF}=171$ hours.

The failure rate for the expected time of failure-free operation according to the above formula is:

$$
\lambda(t)=\frac{e^{z}}{\sigma \cdot t \cdot\left(1+e^{z}\right)}
$$

It follows that:

$$
\lambda(171)=\frac{e^{0,9855}}{4,733 \cdot 171 \cdot\left(1+e^{0,9855}\right)}=8,9974 \cdot 10^{-4} \frac{\text { failure }}{\text { hour }} \cong 8 \frac{\text { failure }}{\text { year of work }}
$$

Also, the unreliability function for the expected time without failure is:

$$
F(t)=1-R(t)=\frac{1}{1+e^{z}}=0,728=72,8 \%
$$

The unreliability function shows that for MTTF $=171$ hours the unreliability is as high as $72.8 \%$. Cumulative Hazard (Cumulative Hazard) is calculated according to the formula:

$$
(T)=\int_{0}^{T} \lambda(t) d=\int_{0}^{T} \frac{e^{Z}}{\sigma \cdot t \cdot\left(1+e^{Z}\right)} d t=\frac{e^{Z}}{\sigma \cdot\left(1+e^{Z}\right)} \cdot \ln (T)
$$

For the value of cumulative risk $\Lambda(T)=0,5$; time $T$ is: $0,154 \cdot \ln (T)=0.5 \rightarrow T$ $=25,7 \mathrm{~h}$. For MTTF $=25.7 \mathrm{~h}$, the unreliability is $64.24 \%$. Given that power plants require a high degree of operational safety, the unreliability should not exceed $30 \%$.

$$
F(t)=1-R(t)=0,3 \rightarrow t \cong 88 h
$$

Given the high required degree of safety of $30 \%$, the required time of preventive maintenance of the filter is $t=88 \mathrm{~h}$ of operation.

\subsection{RESEARCH RESULTS OF THE OF RELIABILITY ANALYSIS OF THE TRANSFORMER CONNECTOR SUBSYSTEM}

Table II. shows the empirical data used for analysis. Fault and duration data are displayed for connector failures in different transformer stations in which the electric connector operates under the similar conditions.

Table II. Empirical data used for analysis

\begin{tabular}{|c|c|c|c|c|c|}
\hline \multirow{2}{*}{$\begin{array}{c}\text { Number } \\
\text { of the } \\
\text { connector }\end{array}$} & \multicolumn{2}{|c|}{$\begin{array}{c}\text { Operating time to failure } \\
\mathrm{K}_{\mathrm{i}}=\mathrm{S}_{\text {Koi }}\end{array}$} & $\begin{array}{c}\text { Logistic time } \\
\text { of corrective } \\
\text { maintenance }\end{array}$ & $\begin{array}{c}\text { Active time } \\
\text { of corrective } \\
\text { maintenance }\end{array}$ & $\begin{array}{c}\text { Duration of } \\
\text { corrective } \\
\text { maintenance }\end{array}$ \\
\cline { 2 - 6 } & $\mathrm{T}_{\text {Ri }}$ [years] & $\mathrm{T}_{\text {Ri }}$ [hours] & $\mathrm{T}_{\text {KLi }}$ [hours] & $\mathrm{T}_{\text {KAi }}$ [hours] & $\mathrm{T}_{\text {Ki }}$ [hours] \\
\hline 1 & 38,64 & 338718 & 8,25 & 1 & 9,25 \\
\hline 2 & 42,45 & 372117 & 9,9 & 1 & 10,9 \\
\hline 3 & 37,28 & 326797 & 4,82 & 1 & 5,82 \\
\hline 4 & 38,66 & 338894 & 0,22 & 0,23 & 0,45 \\
\hline 5 & 29,23 & 256230 & 2,37 & 1 & 3,37 \\
\hline 6 & 23,98 & 210209 & 0,11 & 0,12 & 0,23 \\
\hline 7 & 18,75 & 164363 & 0,45 & 0,45 & 0,9 \\
\hline 8 & 46 & 403236 & 4,82 & 1 & 5,82 \\
\hline 9 & 35,88 & 314524 & 3,17 & 1 & 4,17 \\
\hline 10 & 51,44 & 450923 & 0,75 & 1 & 1,75 \\
\hline 11 & 40,1 & 351517 & 0,44 & 0,44 & 0,88 \\
\hline 12 & 48,83 & 428044 & 2 & 1 & 3 \\
\hline 13 & 44,65 & 391402 & 4,77 & 1 & 5,77 \\
\hline & & & & & \\
\hline
\end{tabular}

Statistical data processing was performed using the Minitab 19 software tool, based on the data from Table II. The results show that the collected empirical data for operating time to failure best follow the Weibull distribution function because for it, the value of the Anderson-Darling test is the lowest, although the log-logistic distribution is very close.

Although the values of the log-logistic Anderson-Darling test is close, the Weibull distribution was selected, and it is used for further calculations.

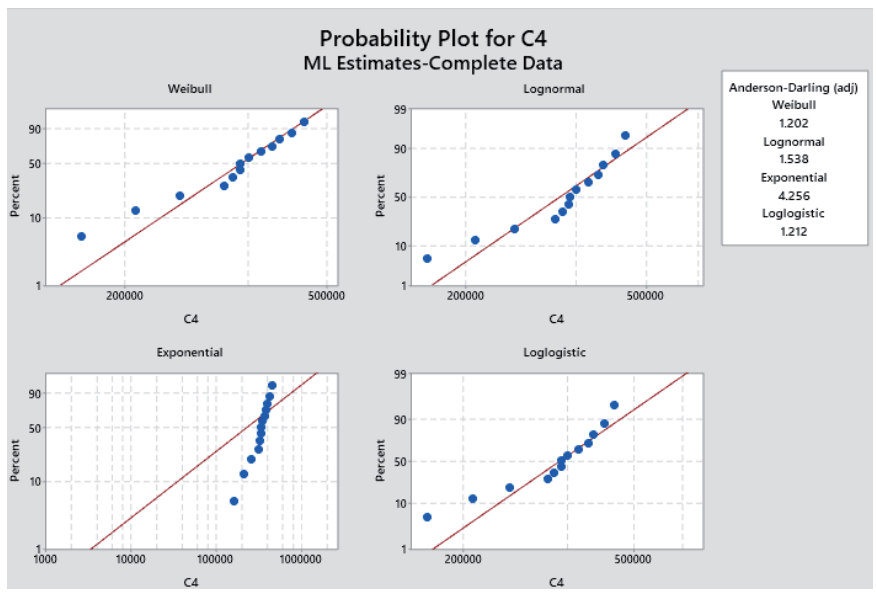

Figure 5. Different distributions for failure probability

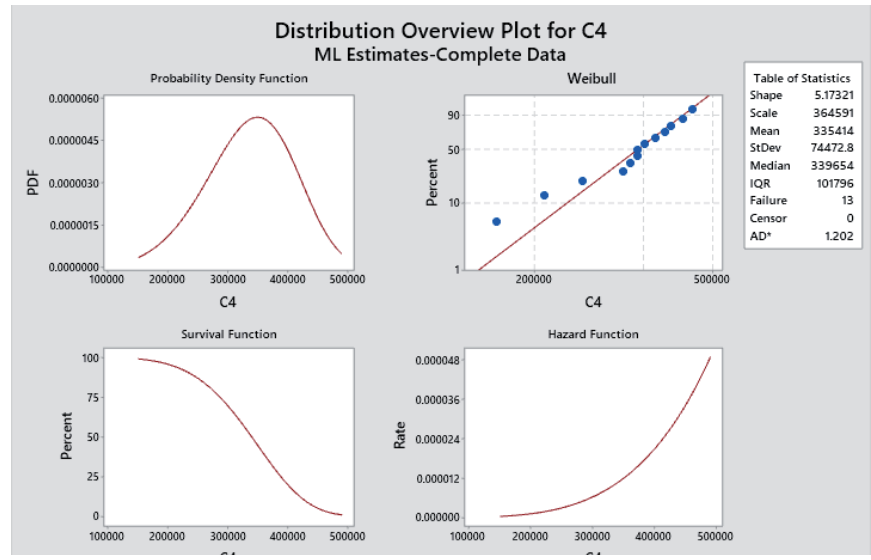

Figure 6. Weibull distribution parameters for failure probability 
The density function of the Weibull distribution is given by the relation:

$$
f(t)=\frac{c}{a} \cdot\left(\frac{t}{a}\right)^{c-1} \cdot e^{-\left(\frac{t}{a}\right)^{c-1}}
$$

where $c$ is shape parameter and $a$ is scale parameter of Weibull distribution.

The values of these parameters were selected based on the results obtained by analysis in Minitab, and they are as follows: $c=5,17321$; $a=$ 364591 . If we insert it in equation (18) we get:

$$
f_{e}(t)=1,419 \cdot 10^{-5} \cdot\left(\frac{t}{364591}\right)^{4.17321} \cdot e^{-\left(\frac{t}{364591}\right)^{5,17321}}
$$

Statistical data processing was repeated with collected data about time for corrective maintenance, logistic time for corrective maintenance and active time of corrective maintenance. The results are shown on Figures 7-12. The results show that the for all three cases collected empirical data best follow the loglogistic distribution function because for it, the value of the Anderson-Darling test is the lowest, although for two cases the Weibul distribution is very close.

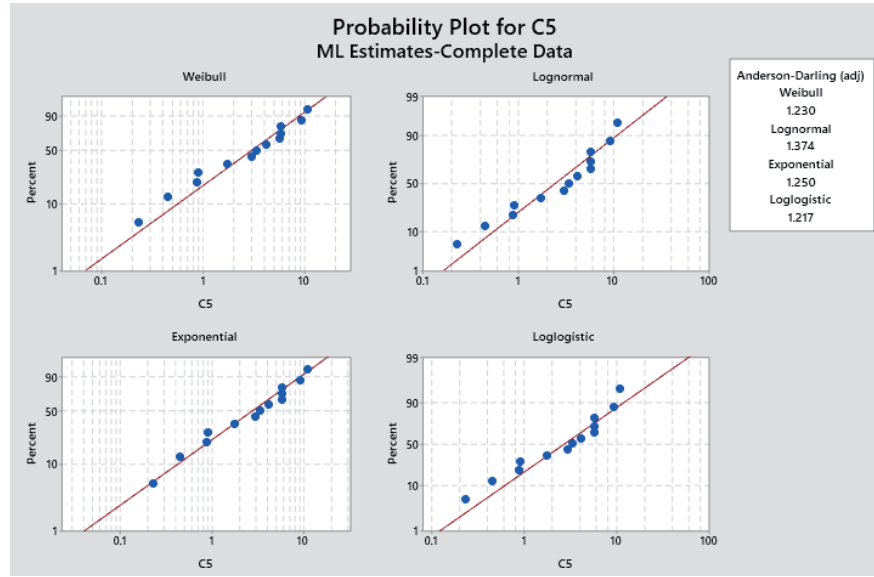

Figure 7. Different distributions for maintenance time probability

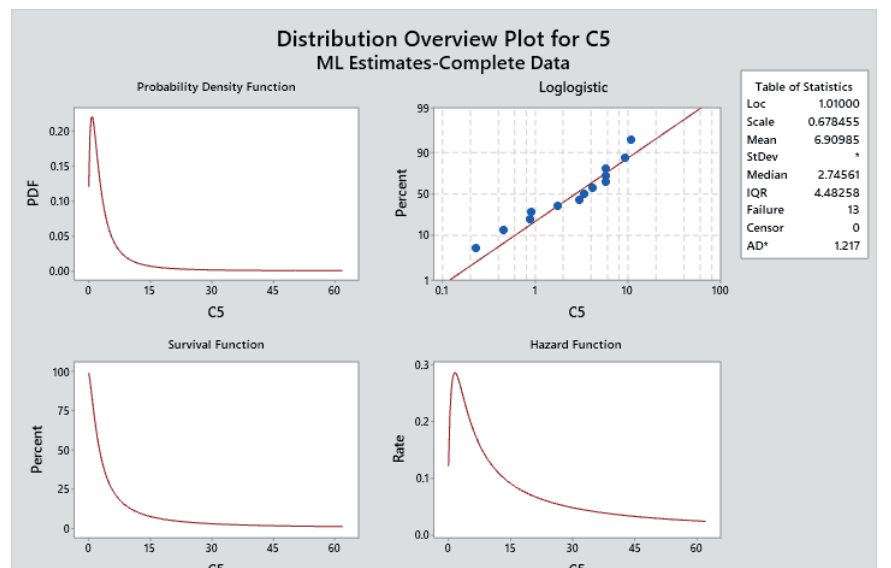

Figure 8. Loglogistic distribution parameters for maintenance time probability

The second statistical processing was made with corrective maintenance times. Figure 8 . shows that the expected logistic time for corrective maintenance of $E_{K}(t)=6,91$ hours.

The third statistical processing was made with logistic corrective maintenance times. Figure 10. shows that the expected logistic time for logistic corrective maintenance of $E_{K L}(t)=9,76$ hours.

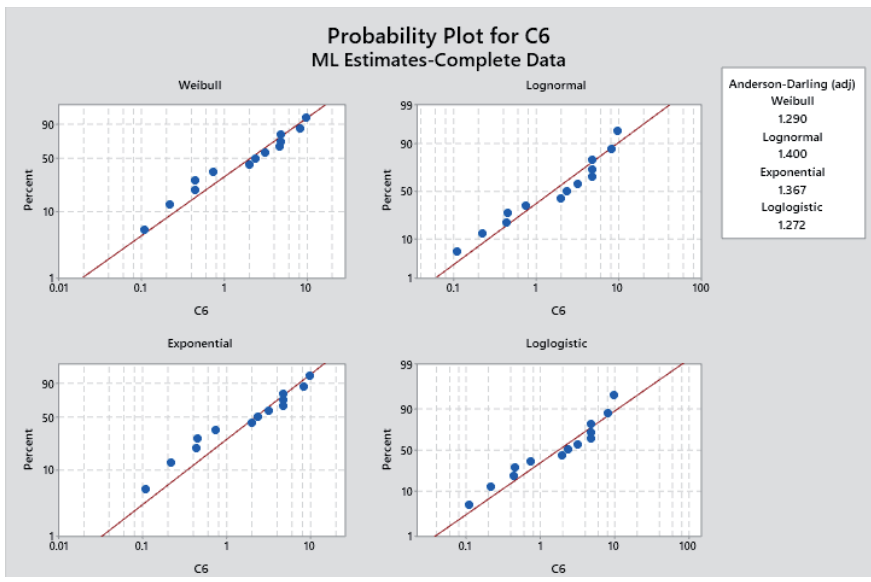

Figure 9. Different distributions for logistic maintenance time probability

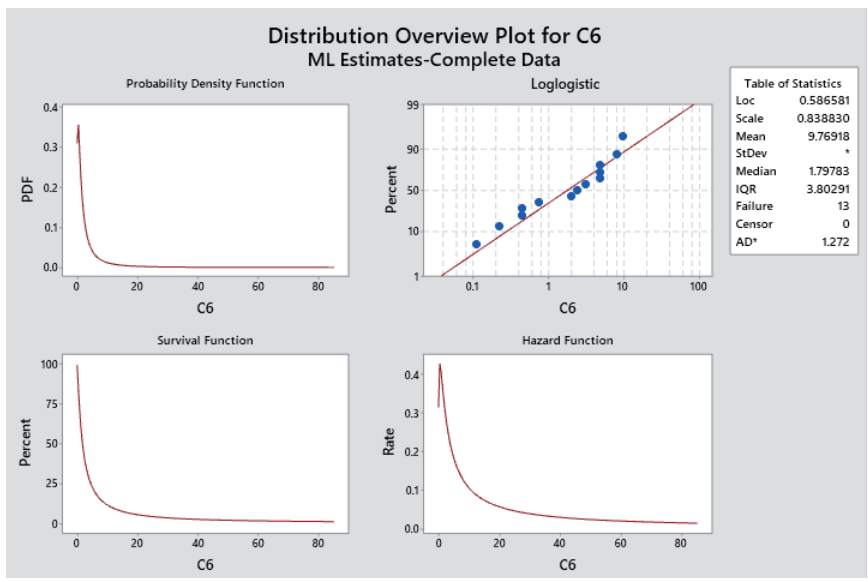

Figure 10. Loglogistic distribution parameters for logistic maintenance time probability

The fourth statistical processing was made with active corrective maintenance times. Figure 12. shows that the expected active time for active corrective maintenance of $E_{K A}(T)=0,95$ hours.

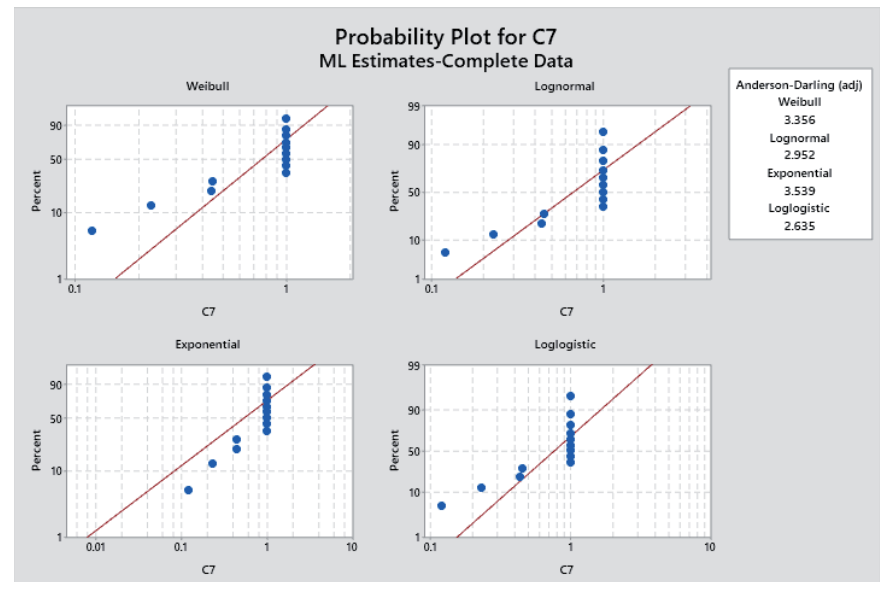

Figure 11. Different distributions for active maintenance time probability 


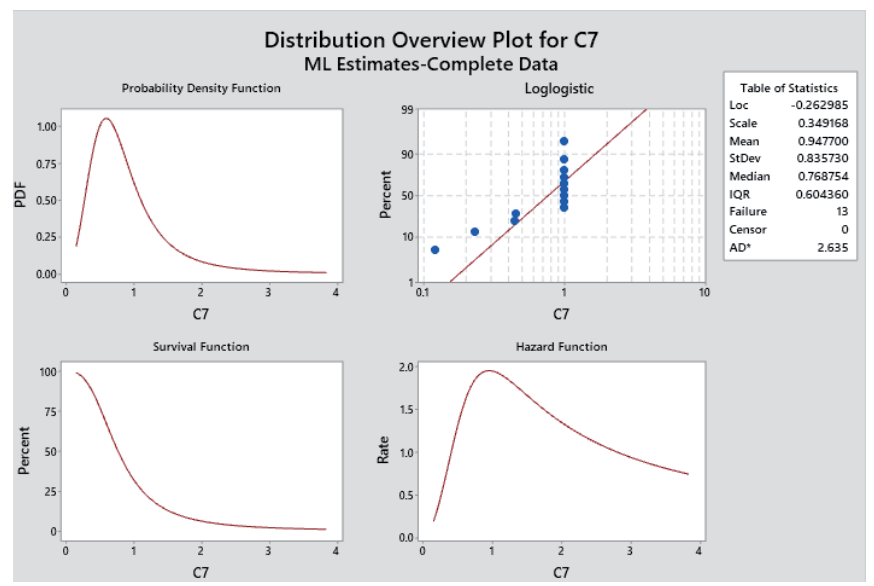

Figure 12. Loglogistic distribution parameters for active maintenance time probability

Table III. shows the numerical models for all four statistical processings.

Table III. Numerical model - presentation of theoretical function of the probability density of characteristic events and the expected time to their of origin

\begin{tabular}{|c|c|c|c|}
\hline $\begin{array}{l}\text { Event } \\
\qquad / \\
\text { times }\end{array}$ & $\begin{array}{l}\text { Theoretical probability } \\
\text { distribution }\end{array}$ & Numerical model (probability density function) & Expected time \\
\hline Fault $\left(K=S_{\mathrm{K} 0}\right)$ & $\begin{array}{c}\text { Twoparameter Weibull } \\
\text { distribution } \\
\text { Shape parameter } \\
\mathrm{C}=5,17321 \\
\text { Scale parameter } \\
\mathrm{a}=364591\end{array}$ & $\begin{array}{c}f_{e}(t)=1,419 \cdot 10^{-5} \cdot\left(\frac{t}{364591}\right)^{4.17321} \\
\cdot e^{-\left(\frac{t}{364591}\right)^{5.17321}}\end{array}$ & $\begin{array}{c}E_{R}(T)= \\
335414 h\end{array}$ \\
\hline $\begin{array}{l}\text { Corrective main- } \\
\text { tenance time, } T_{k}\end{array}$ & $\begin{array}{l}\text { Loglogistic distribution } \\
\text { Location parameter } \mid=1,01 \\
\text { Scale parameter } \\
a=0.678455\end{array}$ & $m_{K}(t)=\frac{e^{\left(\frac{\ln (t)-1,01}{0,678455}\right)}}{t \cdot 0,678455 \cdot\left(1+e^{\left(\frac{\ln (t)-1,01}{0,678455}\right)}\right)^{2}}$ & $E_{K}(T)=6,91 h$ \\
\hline $\begin{array}{l}\text { Logistic correcti- } \\
\text { ve maintenance } \\
\text { time, } \mathrm{T}_{\mathrm{KL}}\end{array}$ & $\begin{array}{l}\text { Loglogistic distribution } \\
\text { Location parameter } \\
\text { l=0,586581 } \\
\text { Scale parameter } \\
a=0,838830\end{array}$ & $m_{K L}(t)=\frac{e^{\left(\frac{\ln (t)-0,586581}{0,838830}\right)}}{t \cdot 0,838830 \cdot\left(1+e^{\left(\frac{\ln (t)-0,586581}{0,838830}\right)}\right)^{2}}$ & $E_{K L}(T)=9,76 h$ \\
\hline $\begin{array}{l}\text { Active corrective } \\
\text { maintenance } \\
\text { time, } T_{K A}\end{array}$ & $\begin{array}{c}\text { Loglogistic distribution } \\
\text { Location parameter l=- } \\
0,262985 \\
\text { Scale parameter } \\
a=0,349168\end{array}$ & $m_{K A}(t)=\frac{e^{\left(\frac{\ln (t)+0.2629851}{0.349168}\right)}}{t \cdot 0,349168 \cdot\left(1+e^{\left(\frac{\ln (t)+0.262985}{0.349168}\right)}\right)^{2}}$ & $E_{K A}(T)=0,95 h$ \\
\hline
\end{tabular}

If we insert times for corrective maintance $E_{k A}(T)$ and $E_{k}(T)$ from Table III. into relations (6) and (7), we get costs of preventive maintance $3899,5 \mathrm{kn}$ and costs of corrective maintenance $6304,18 \mathrm{kn}$.

If we insert costs of preventive and corrective maintance into relations (4) and (5), we will get relative costs of preventive maintance $=38,2$ and relative costs of corrective maintenance.

In order to determine the optimal point for preventive replacement of the transformer connector subsystem, it is necessary to determine the function of relative maintenance costs.

The operational reliability $R_{e}(t)$ is described by the relation (10). If we insert the function of the probability density of the fault $f_{e}(t)$ from Table III. in rela-

$$
R_{e}(t)=1-\int_{0}^{t} 1,419 \cdot 10^{-5} \cdot\left(\frac{t}{364591}\right)^{4,17321} \cdot e^{-\left(\frac{t}{364591}\right)^{5,17321}} d t
$$

By integrating relation (20) we obtain the operational reliability of the transformer connector subsystem:

$$
R_{e}(t)=e^{-\left(\frac{t}{364591}\right)^{5.17321}}
$$


By inserting parameters of maintenance distribution and costs as well as operational reliability of the transformer connector subsystem into the relation (3) we get the function of the relative costs of maintenance for transformer connector subsystem:

$$
c_{u, R e, \text { rel }}\left(T_{R}\right)=0,382 \cdot \frac{1}{T_{R}}+0,618 \cdot \frac{T_{R}-\int_{0}^{T_{R}} e^{-\left(\frac{t}{364591}\right)^{5.17321}} d t}{T_{R} \cdot \int_{0}^{T_{R}} e^{-\left(\frac{t}{364591}\right)^{5.17321}} d t}
$$

A graph of the function of relative maintenance costs was drawn in program Mathcad 15 and shown in Figures 13., 14.

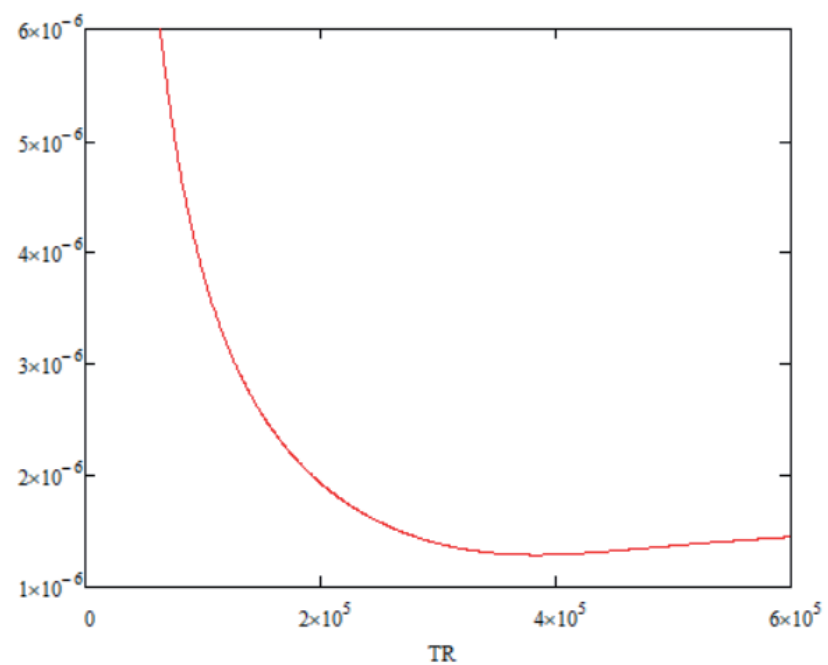

Figure 13. Graph of the function of relative maintenance costs cu,Re,rel(TR) for MV transformer connector subsystem

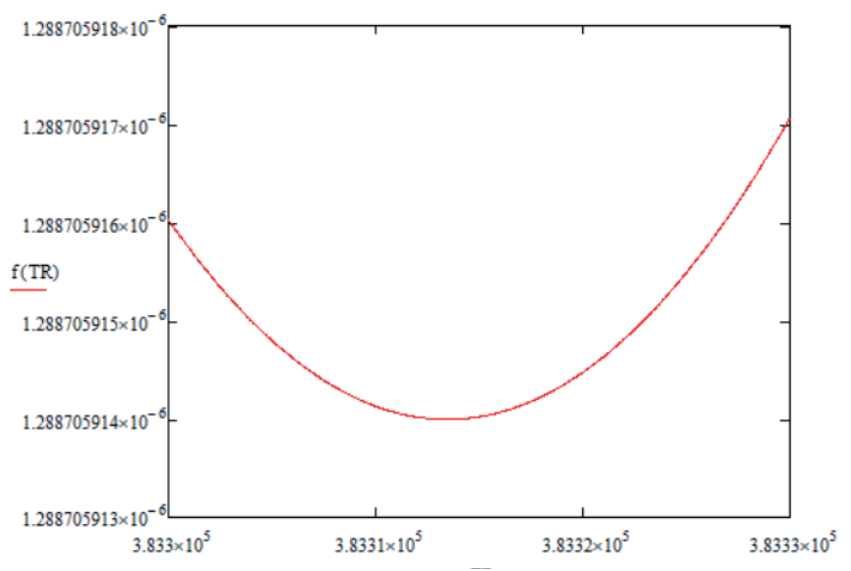

Figure 14. Minimum of the function of relative maintenance costs cu,Re,rel(TR) for MV transformer connector subsystem
Using the program Mathcad 15, the minimum of the function shown in Figure 14. was determined. In accordance with the obtained minimum, the optimal time of preventive maintenance of the medium voltage transformer connector subsystem is obtained. The optimal time for preventive maintenance is 383313 hours or 43,73 years.

\section{CONCLUSIONS}

First part of the article presents the results of the analysis performed on the empirical data of filter failure at the gas turbine compressor intake, obtained from the power plant in HEP's portfolio. The Minitab 19 software package was used for the analysis, and a three-parameter log-normal distribution was determined with a high level of significance, and the hypothesis was accepted. The expected uptime of the MTTF and the required time of preventive maintenance of the filter in relation to the required safety were also determined. The results obtained by the analysis can be used as a basis for making decisions related to the maintenance strategy, in order to prevent or minimize plant downtime caused by failure and thus losses. As said in the introduction of this paper, when the compressor suction filter is damaged or dirty, the power plant operation must be stopped and the filter replaced, so the aim of this paper is to develop a model of preventive maintenance, in order to be able to predict the potential failure of the filter based on empirical data and to prevent the sudden exit of the power plant from the operation. Data used for these calculations were collected during an extensive period of time, and they are empirical, i.e. they were collected from one of the powerplants from the HEP portfolio. Results of the analysis shows that the required time of preventive maintenance of the filter is $t=88 \mathrm{~h}$ of operation. That means that after 88 hours of operation of the powerplant, there is a good chance that the malfunction will occur on the filter. Knowing that, filters can be purchased in advance and kept in stock, and speed up the return of the power plant to operation, or reduce financia losses due to production downtime. Second part of the article presents the results of the analysis of the empirical data performed in order to determine optimal time for preventive maintenance of the medium voltage transformer connector subsystem. The results obtained by the analysis can be used as a basis for making decisions related to the maintenance strategy in order to prevent the sudden electric power failure that would left consumers without electricity and cause financial losses to electric energy seller. The analysis showed that the optimal time for preventive maintenance of transformer connector subsystem for the observed case is 43,73 years, which means that this is not a failure that happens often, and therefore it is not necessary keeping of spare parts in stock, or monitoring this part of equipment particularly. As shown in this paper, reliability analysis is very useful for determing time period after which a failure may occur. Knowing that time period is very important because it allows preparation for failure (planned maintenance, keeping spare parts in stock, etc.), which can significantly reduce the financial losses causes by failure.

\section{REFERENCES}

[1] J. Batelić: Contribution to increasing the reliability of the $\mathrm{m}$ system of TPP Plomin 2 / doctoral thesis, Rijeka, Faculty of Engineering, 2019

[2] D. Matika, J. Batelić: Determining the operational reliability of the Plomin 2 thermal power plant for the purpose of evaluating critical national infrastructure; III. International scientificprofessional conference: "New security threats and critical national infrastructure", pp. 218227.UDK 351.751: 504, Zagreb 2013

[3] J. Batelić, D. Matika, K. Griparić: Maintenance of hydraulic cylinders based on the achieved level of operational reliability, Polytechnic and design, Vol. 7 No. 4, str. 281-288, 2019.
[4] A. Birolini: Reliability Engineering, Theory and Practice, Fifth edition, Springer, 2006.

[5] Minitab software tool: https:// support.minitab.com/en-us/ minitab/18/help-and-how-to/ statistics/basic-statistics/ statistics/basic-statistics/ supporting-topics/normality/ the-anderson-darling-statistic/

[6] Mathcad software tool: https:// www.mathcad.com/en/

[7] R. E. Barlow: Engineering Reliability, Society for Industria and Applied Mathematics, University of California, Berkeley, 1998.

[8] B. S. Dhillon: Reliability engineering in systems design and operation, Van Nostrand Reinhold 1983.

[9] T. R. Chandrupatla: Quality and
Reliability in Engineering, 1st edition, Cambridge University Press, 2009.

[10] A. J. Hallinan Jr.: A Review of the Weibull Distribution, Journa of Quality, Volume 25, 1993 ssue 2, Pages 85-93, Published Online: 21 Feb 2018

[11] A. A. AlShomrani, A. I. Shawky, O. H. Arif, M. Aslam: Log-logistic distribution for survival dana analysis using MCMC, SpringerPlus 5, 1774 (2016)

[12] C. Stenström, P. Norrbin, A. Parida, U. Kumar: Preventive and corrective maintenance - cost comparison and cost-benefit analysis, Structure and Infratructur, Enginering, Vnfra12, 2016 - Issue 5, Pages 603 12, 2016 - Issue 5, Pages 603617, Published online: 13 May
[13] C. D. Lai, G. D. Lin: Mean time to failure of systems with dependent components, Applied Mathematics and Computation, Volume 246, Pages 103-111, November 2014.

[14] T. W. Anderson: Anderson-Darling Tests of Goodness-of-Fit, International Encyclopedia of Statistical Science, Springer, Berlin, Heidelberg, 2011.

[15] C. C. Chang: Optimum preventive maintenance policies for systems subject to random working times, replacement, and minimal repair, Computers \& Industrial Engineering, Volume 67, Pages 185-194, January 2014. 